\title{
Systemic Lupus Erythematosus With Lupus Nephritis Presented With Recurrent Massive Ascites: A Case of Pseudo-Pseudo Meigs Syndrome
}

\author{
Abeer AWAD $(D$, , Mervet ESSAM $[$, Asmaa EZZAT $@$, Manal EL MENYAWI@ \\ Department of Internal Medicine, Kasr Al Ainy, Cairo University, Cairo, Egypt
}

Systemic lupus erythematosus (SLE) is an autoimmune multi-systemic disease with heterogeneous manifestations. It is well known that polyserositis is common in SLE patients, particularly pleural and pericardial affection; however, peritoneal affection is not so common. ${ }^{1}$ This should raise our suspicions to exclude other causes of ascites in SLE patients particularly if ascites is recurrent or massive.

In this article, we report the case of a 43-year-old female patient with SLE with lupus nephritis diagnosed 15 years ago based on clinical findings including musculoskeletal and renal manifestations, positive antinuclear antibodies and anti-double stranded deoxyribonucleic acid with consumed complement 3 and 4. Patient was prescribed hydroxychloroquine, steroids, cyclophosphamide and mycophenolate mofetil. She presented to us with lupus activity in form of autoimmune hemolytic anemia with a history of recurrent massive ascites and bilateral pleural effusion. The condition prompted us to search for the causes of refractory ascites which might be malignant or infectious, or tuberculosis (TB) in particular, after ruling out renal, cardiac, hepatic etiologies. A written informed consent was obtained from the patient.
We performed extended investigations that showed normal blood albumin level. Tumor markers were significant only for an elevated serum cancer antigen 125 (CA-125) (80 U/L normal <35). Work-up for TB was negative including adenosine deaminase and polymerase chain reaction in ascitic sampling. Cytology was negative for malignant cells. We conducted computed tomography scan for the chest, abdomen and pelvis with contrast showing massive ascites with ill-defined omental thickening related to the anterior abdominal wall. A cystic lesion was seen at perineal region, minimal bilateral pleural effusion and massive abdominal ascites. Laparotomy and biopsy were performed revealing fat necrosis. Subsequently, magnetic resonance imaging of the abdomen and pelvis was conducted but it was insignificant apart from massive ascites with adhesions. No ovarian masses were observed (Figure 1). In lower perineal region, herniating peritoneal sac was noted containing ascitic fluid representing peritoneocele (Figure 2).

The patient was started on $1 \mathrm{~g} /$ day pulse methylprednisolone therapy for three days with partial improvement in hemoglobin level. At this point, rituximab was started particularly since the patient presented with refractory

\footnotetext{
Received: May 27, 2018 Accepted: November 08, 2018 Published online: January 08, 2019

Correspondence: Abeer Awad, MD. Department of Internal Medicine, Kasr Al Ainy, Cairo University, 11562 Cairo, Egypt. Tel: 00201002776494 e-mail: beero4a@yahoo.com 


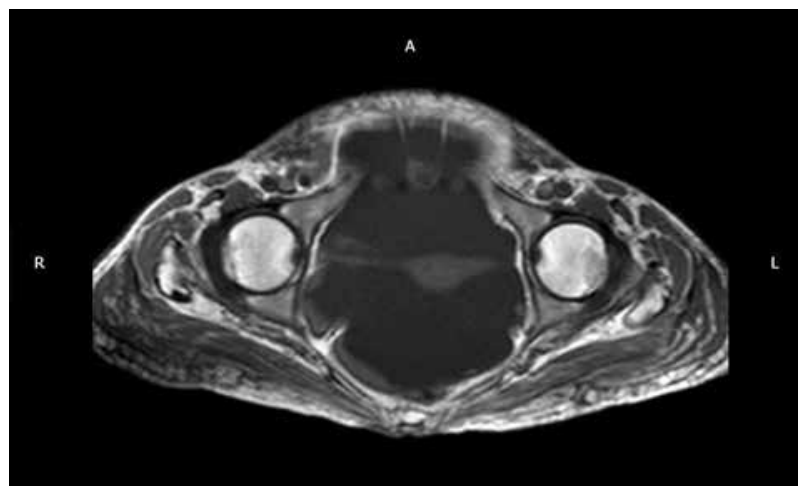

Figure 1. Magnetic resonance imaging of abdomen and pelvis showing massive ascites with adhesions (normal ovaries).

manifestations of lupus in spite of her long-term compliance on immunosuppressive drugs. Along with the short-term effect of rituximab, the patient improved markedly in terms of anemia, creatinine and general condition; yet, ascites was moderately ameliorated. Unfortunately, the patient was lost to follow-up; therefore, the long-term effect could not be traced.

The constellation of recurrent massive ascites, bilateral pleural effusion together with an elevated CA-125 in absence of ovarian malignancy led to a diagnosis of pseudo-pseudo Meigs Syndrome (PPMS), a rare manifestation of SLE. A differential diagnosis should be performed for PPMS in any SLE case with massive ascites after exclusion of malignancy to avoid unnecessary invasive investigations.

Elevated CA-125 in PPMS may be explained by this type of tumor marker that is highly expressed in mesenchymal cells, produced by cytokines activation as vascular endothelial growth factor or fibroblast growth factor, thereby irritating the mesothelial cells. ${ }^{2}$ CA-125 is presumed to be an inflammatory marker. ${ }^{3}$ The definite inciting agents that modulate this inflammatory stream might be an operation ${ }^{4}$ or drug as leflunomide. ${ }^{5}$ However, our case had no obvious risk factor and it is likely that uremic toxins are potentially pro-inflammatory targeting different cytokines (interleukin [IL]-1 beta, IL-18, IL-6, tumor necrosis factor alpha). ${ }^{6}$ Thus, we hypothesize that renal impairment and uremia of our patient might have increased the cytokines level exacerbating the inflammatory process and rapid reaccumulation of ascites. In this article, we portrayed a case of

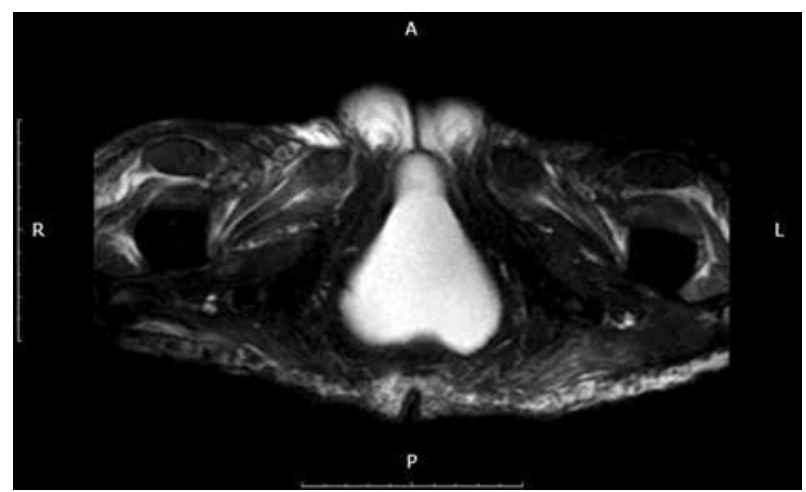

Figure 2. Magnetic resonance imaging of abdomen and pelvis showing herniated peritoneal sac containing ascitic fluid representing peritoneocele.

lupus nephritis complicated with renal impairment presenting with a benign rare feature of SLE, PPMS.

\section{Declaration of conflicting interests}

The authors declared no conflicts of interest with respect to the authorship and/or publication of this article.

\section{Funding}

The authors received no financial support for the research and/or authorship of this article.

\section{REFERENCES}

1. Zampeli E, Skopouli FN, Moutsopoulos HM. Polyserositis in a patient with active systemic lupus erythematosus: a case of pseudo-pseudo meigs syndrome. J Rheumatol 2018;45:877-8.

2. Torres Jiménez AR, Solís-Vallejo E, Céspedes-Cruz AI, Zeferino Cruz M, Rojas-Curiel EZ, SánchezJara B. Tjalma syndrome (pseudo-pseudo Meigs') as initial manifestation of juvenile-onset systemic lupus erythematosus. Reumatol Clin 2017 May 15. pii: S1699-258X(17)30091-8. [Epub ahead of print]

3. Dalvi SR, Yildirim R, Santoriello D, Belmont HM. Pseudo-pseudo Meigs' syndrome in a patient with systemic lupus erythematosus. Lupus 2012;21:1463-6.

4. Abramov Y, Anteby SO, Fasouliotis SJ, Barak V. The role of inflammatory cytokines in Meigs' syndrome. Obstet Gynecol 2002;99:917-9.

5. Ural UM, Kiliç A, Güngör T, Ozdal B, Mollamahmutoğlu L. Tjalma's or pseudo-pseudo-Meigs' syndrome: a case report. Clin Exp Dermatol 2008;33:363-4.

6. Bes C, Soy M. Pseudo-pseudo Meigs syndrome developed under the leflunomide therapy. Rheumatol Int 2011;31:521-3. 